\title{
Ensino da reanimação neonatal em maternidades públicas das capitais brasileiras
}

\author{
Teaching neonatal resuscitation at public hospitals in Brazilian state capitals \\ Maria Fernanda B. de Almeida1, Ruth Guinsburg'2, José Orleans da Costa3, \\ Lêni Márcia Anchieta4, Lincoln M. Silveira Freire5, \\ Pesquisadores do Programa de Reanimação Neonatal da Sociedade Brasileira de Pediatria 6
}

\section{Resumo}

Objetivo: Analisar o ensino da reanimação neonatal para médicos e enfermeiros em formação e para residentes em pediatria e neonatologia em maternidades públicas brasileiras.

Métodos: Estudo transversal multicêntrico, realizado em 36 maternidades de 20 capitais brasileiras em junho de 2003. O coordenador local preencheu questionário com dados referentes à realização de ensino específico da reanimação neonatal e atuação em sala de parto de alunos de Medicina e de Enfermagem, residentes de Pediatria e de Neonatologia. Os dados foram analisados de forma descritiva.

Resultados: Das 36 maternidades, 23 recebiam alunos de Medicina: em 13, eles atuavam na sala de parto; 12 ofereciam ensino específico da reanimação e, em duas das 13 maternidades com internos atuando na sala de parto, não era oferecido treinamento em reanimação. Das 36 maternidades, 23 recebiam alunos de Enfermagem: em oito, os alunos atuavam na sala de parto. Apenas uma oferecia ensino, e nas outras sete não havia qualquer treinamento. Das 36 maternidades, 27 tinham residentes de Pediatria, que atuavam na sala de parto e recebiam treinamento em reanimação neonatal (teórico com 2-3 horas em quatro maternidades e teórico-prático com 4-64 horas em 23). Das 36 maternidades, 15 recebiam residentes em Neonatologia, que atuavam na sala de parto e recebiam treinamento em reanimação neonatal (teórico de 2 horas em uma e teórico-prático com 3-68 horas em 14).

Conclusão: $O$ ensino de reanimação por meio de cursos formais é insuficiente para os alunos de graduação em Medicina e Enfermagem e heterogêneo para os residentes em Pediatria e em Neonatologia.

J Pediatr (Rio J). 2005;81(3):233-9: Ressuscitação cardiopulmonar, educação, recém-nascido.

\begin{abstract}
Objective: To analyze the teaching of neonatal resuscitation offered by Brazilian public hospitals to undergraduate doctors and nurses, pediatric residents and neonatal fellows.

Methods: This cross-sectional multicenter study included 36 hospitals in 20 Brazilian state capitals during June/2003. Local coordinators collected data regarding what the institutions offer to undergraduate doctors and nurses, pediatric residents and neonatal fellows in terms of neonatal resuscitation training and practical activities in neonatal delivery room care. Descriptive analysis was performed.
\end{abstract}

Results: Twenty-three of the 36 institutions had undergraduate doctors: at 13 of them students were given practical activities in the delivery room, 12 offered neonatal resuscitation training, and at two of the 13 hospitals, interns cared for neonates in the delivery room without specific training. Twenty-three of the 36 hospitals had undergraduate nurses: at eight of them students were given practical activities in the delivery room and at seven of them nursing students cared for neonates in the delivery room without specific training. Twenty-seven of the 36 institutions had pediatric residence programs: at all of them the trained residents cared for neonates in the delivery room, but this training was heterogeneous: theoretical training (2-3 hours) at four institutions and theoretical and practical training (4-64 hours) at 23. Additionally, 15 had neonatal fellowship programs: at all of these the trained fellows cared for neonates in the delivery room, but this training was heterogeneous: theoretical training ( 2 hours) at one hospital and theoretical and practical training (3-68 hours) at 14 .

Conclusion: Formal neonatal resuscitation training is insufficient during medical and nursing graduation and heterogeneously offered to pediatric residents and neonatal fellows.

J Pediatr (Rio J). 2005;81(3):233-9: Cardiopulmonary resuscitation, education, infant, newborn.

1. Professora adjunta, Universidade Federal de São Paulo (UNIFESP), Escola Paulista de Medicina (EPM). Responsável por fomentos em pesquisa, Programa de Reanimação Neonatal, Sociedade Brasileira de Pediatria (SBP) na gestão 2001-2003.

2. Professora adjunta e livre-docente, UNIFESP, EPM. Responsável por fomentos em pesquisa, Programa de Reanimação Neonatal SBP na gestão $2001-2003$.

3. Coordenador do Programa de Reanimação Neonatal SBP, na gestão 2001-2003.

4. Coordenadora adjunta, Programa de Reanimação Neonatal SBP, na gestão 2001-2003.

5. Presidente da SBP na gestão 2001-2003.

6. Pesquisadores do Programa de Reanimação Neonatal da SBP (relação completa no final do artigo).

Artigo submetido em 03.11.04, aceito em 12.01.05.

Como citar este artigo: de Almeida MF, Guinsburg R, da Costa JO, Anchieta LM, Freire LM, Pesquisadores do Programa de Reanimação Neonatal da Sociedade Brasileira de Pediatria. Ensino da reanimação neonatal em maternidades públicas das capitais brasileiras. J Pediatr (Rio J). 2005;81:233-9. 


\section{Introdução}

No Brasil, o número de nascidos vivos em 2001 foi de 3.106.525, sendo que 61.807 deles morreram antes de completar 1 ano de idade ${ }^{1,2}$. O número de óbitos até 7 e 28 dias de vida foi, respectivamente, 31.566 e 8.639 naquele ano, com taxas de mortalidade neonatal precoce e tardia de 14,0 e 3,8 por 1.000 nascidos vivos ${ }^{1,2}$. Durante toda a última década, a asfixia perinatal foi a principal causa de morte em cerca de $10 \%$ dos óbitos neonatais ${ }^{3}$, sem levar em conta a contribuição dos processos asfíxicos na mortalidade neonatal por outras causas. Estima-se que os dados aqui colocados correspondam a cerca de $80 \%$ do número total de óbitos de recém-nascidos no país, pois os dados de mortalidade infantil e neonatal são subestimados, devido à notificação incompleta, principalmente na região Norte e Centro-Oeste ${ }^{3}$.

A assistência adequada ao recém-nascido na sala de parto é fundamental para prevenir o aparecimento das lesões asfíxicas, que levam ao óbito neonatal. Dentre as oito intervenções estratégicas para diminuir a mortalidade de crianças até 5 anos de idade em nível global, a reanimação ao nascer tem papel de destaque, estimando-se que a aplicação dos procedimentos necessários possa prevenir, a cada ano, a morte de 359.000 recém-nascidos em todo o mundo ${ }^{4}$. Além disso, a reanimação neonatal rápida e efetiva pode, nos sobreviventes, reduzir a presença de seqüelas neurológicas, que acarretam prejuízos para a qualidade de vida da criança e de sua família, além de elevados custos para a sociedade, incluindo os gastos com a saúde e a produtividade do indivíduo afetado.

Vários estudos mostram que o treinamento em reanimação constitui-se em estratégia relativamente simples e não-onerosa para diminuir a mortalidade neonatal precoce ${ }^{4-8}$. Dentre as várias maneiras de aperfeiçoar o conhecimento dos profissionais no tema, a mais bem sucedida é a proposta pelo Comitê Internacional de Reanimação Neonatal ${ }^{9,10}$. Esse programa educacional foi delineado para ensinar de forma seqüencial a abordagem ao recém-nascido que precisa ser reanimado nos minutos críticos do pós-parto, contando com um Manual de Reanimação Neonatal que já foi traduzido para 27 diferentes idiomas ${ }^{11}$.

No Brasil, o Programa de Reanimação Neonatal foi iniciado em 1994 pela Sociedade Brasileira de Pediatria e, ao final de 1997, praticamente todos os estados já possuíam instrutores aptos a multiplicar os conhecimentos relativos ao atendimento do recém-nascido na sala de parto. As aulas focalizam os passos iniciais da reanimação neonatal, a ventilação com balão e máscara, a massagem cardíaca, a intubação traqueal e o uso de medicações em sala de parto. O material didático é apresentado em português, e o Manual de Reanimação Neonatal, edições originais de 1990, 1994 e 2000, foi traduzido para o português em 1994, 1996 e 2002 , respectivamente ${ }^{12}$. Atualmente, a Sociedade Brasileira de Pediatria conta com 388 instrutores pediatras, que até o final de 2003 já proporcionaram 1.123 cursos de treinamento teórico-prático em reanimação neonatal para cerca de 24.000 profissionais de saúde de nível superior que atuam em sala de parto ${ }^{13}$.
Nesse contexto, o presente estudo visou traçar o perfil do ensino da reanimação neonatal em sala de parto de maternidades públicas das capitais brasileiras no ano de 2003. Dentre os objetivos específicos, destacam-se:

- Verificar quais maternidades eram local de ensino para alunos de graduação em Medicina e Enfermagem, para residentes em Pediatria e para residentes e/ou especializandos em Neonatologia.

- Analisar as características do treinamento em reanimação neonatal para as diversas categorias de alunos nessas instituições.

- Avaliar o número de instituições nas quais as diversas categorias de profissionais em treinamento participam do atendimento ao recém-nascido na sala de parto.

\section{Métodos}

Estudo epidemiológico observacional, com coleta de dados transversais relacionados ao ensino da reanimação neonatal em sala de parto nos vários centros envolvidos, no período de 01 a 30 de junho de 2003. A coleta de dados foi iniciada após aprovação do projeto pelo Comitê de Ética em Pesquisa da instituição vinculada aos pesquisadores principais e da Diretoria Clínica de cada uma das instituições participantes.

Foram incluídas maternidades públicas das capitais brasileiras que tivessem registrado, no mínimo, 5.000 nascidos vivos no ano 2000, segundo informação do Ministério da Saúde ${ }^{1}$. Cada uma das cinco regiões do Brasil esteve representada por, no mínimo, $1 \%$ e, no máximo, $4 \%$ dos seus nascidos vivos. A pesquisa foi realizada em 20 capitais e incluiu 36 instituições nas seguintes regiões: Norte (uma em Manaus e duas em Belém), Nordeste (uma em Teresina, Natal, Recife, Maceió, Aracajú e Salvador, duas em São Luís e três em Fortaleza), Sudeste (uma em Vitória, duas em Belo Horizonte, quatro no Rio de Janeiro e sete em São Paulo), Sul (uma em Florianópolis e duas em Curitiba e Porto Alegre) e Centro-Oeste (uma em Brasília, Campo Grande e Goiânia). Em São Paulo, Rio de Janeiro e Porto Alegre não foram incluídos hospitais universitários federais ou estaduais. Em cada maternidade, um pediatra foi convidado a coordenar localmente o estudo. Cada um desses pesquisadores coletou os seguintes dados:

\section{Caracterização das instituições estudadas}

Pesquisou-se a esfera administrativa do hospital; a caracterização do hospital como geral ou só maternoinfantil; o número de leitos de alojamento conjunto, de cuidados intermediários e intensivos, classificados segundo as portarias do Ministério da Saúde; a caracterização da população de recém-nascidos atendida em cada instituição nos meses de janeiro a junho de 2003; o número de salas de parto em cada instituição e de mesas de reanimação, com recursos materiais mínimos 14,15 para o atendimento ao recém-nascido e o número total de pediatras e enfermeiros com participação no atendimento ao recém-nascido em sala de parto. 


\section{Ensino de reanimação neonatal}

Caracterização da instituição como local de ensino para graduação em Medicina (quinto ou sexto ano) e Enfermagem (terceiro ou quarto ano), para residência médica em Pediatria (R1 e R2) e para especialização ou residência em Neonatologia.

Existência de treinamento formal em reanimação neonatal para alunos de graduação em Medicina e Enfermagem e para residentes e especializandos em Pediatria e Neonatologia, considerado como ausente ou somente teórico (número de horas), ou teórico-prático (número de horas) em cada instituição.

Oferta pelas instituições de campo prático de treinamento, com presença dos alunos, residentes e especializandos no atendimento ao recém-nascido nas salas de parto.

Os dados foram anotados em questionários impressos por cada pesquisador local e enviados por correio para as pesquisadoras principais. A seguir, codificou-se a resposta à cada pergunta, de maneira a criar uma planilha que minimizasse os erros de digitação. Essa planilha foi criada no Programa Microsoft Access ${ }^{\circledR}$. Após checagem da homogeneidade da entrada dos dados nas planilhas, procedeu-se à análise descritiva por meio do programa SPSS 10.0. Os resultados qualitativos estão descritos em número e porcentagem de eventos, e as quantitativas por medidas de tendência central (média e/ou mediana) e de variação (erro padrão ou valores mínimos e máximos). Analisou-se os dados em conjunto, sem identificação das cidades e maternidades envolvidas ou dos profissionais de saúde de cada local.

\section{Resultados \\ Caracterização das instituições estudadas}

Em relação às características gerais das maternidades estudadas, observa-se que a maioria pertencia à esfera pública estadual, com mais de $90 \%$ dos pacientes atendidos pelo Sistema Único de Saúde. Cerca de metade delas localizava-se em hospitais gerais, dois terços participavam do Programa Iniciativa Amigo da Criança, sendo a maioria referência para gestação de risco. Em média, cada instituição contava com 54 leitos de alojamento conjunto, 21 de cuidados intermediários e 12 de intensivos. O número mediano mensal de nascidos vivos de janeiro a junho de 2003 por instituição foi 365, variando de 285 a 466. A população de nascidos vivos das maternidades caracterizou-se por ser de muito baixo peso em $3 \%$ e de baixo peso em $15 \%$. Os pediatras que atuavam no atendimento ao recém-nascido na sala de parto eram predominantemente plantonistas. Havia, em média, quatro enfermeiras por instituição, em cada turno, envolvidas no cuidado neonatal ao nascimento. As características específicas em cada região analisada encontram-se na Tabela 1.

Tabela 1 - Características gerais das 36 instituições, de acordo com a região geográfica

\begin{tabular}{|c|c|c|c|c|c|c|}
\hline & $\begin{array}{c}N \\
n=3\end{array}$ & $\begin{array}{c}\text { NE } \\
n=11\end{array}$ & $\begin{array}{c}\text { SE } \\
n=14\end{array}$ & $\begin{array}{c}S \\
n=5\end{array}$ & $\begin{array}{c}\text { Co } \\
n=3\end{array}$ & $\begin{array}{c}\text { Total } \\
n=36\end{array}$ \\
\hline $\begin{array}{l}\text { Hospital federal } \\
\text { estadual } \\
\text { municipal } \\
\text { privado }\end{array}$ & $\begin{array}{l}0 \\
3 \\
0 \\
0\end{array}$ & $\begin{array}{l}4 \\
7 \\
0 \\
0\end{array}$ & $\begin{array}{l}1 \\
4 \\
8 \\
1\end{array}$ & $\begin{array}{l}1 \\
1 \\
0 \\
3\end{array}$ & $\begin{array}{l}0 \\
1 \\
1 \\
1\end{array}$ & $\begin{array}{c}6(17 \%) \\
16(44 \%) \\
9(25 \%) \\
5(14 \%)\end{array}$ \\
\hline Hospital geral & $\begin{array}{l}2 \\
1\end{array}$ & $\begin{array}{l}5 \\
6\end{array}$ & $\begin{array}{l}7 \\
7\end{array}$ & $\begin{array}{l}3 \\
2\end{array}$ & $\begin{array}{l}2 \\
1\end{array}$ & $\begin{array}{l}19(53 \%) \\
17(47 \%)\end{array}$ \\
\hline Iniciativa Amigo da Criança & 2 & 8 & 7 & 5 & 2 & $24(67 \%)$ \\
\hline Referência de gestação de risco & 3 & 11 & 12 & 4 & 2 & $32(89 \%)$ \\
\hline $\begin{array}{l}\text { Leitos/Instituição * } \\
\text { Alojamento conjunto } \\
\text { Cuidados intermediários } \\
\text { Cuidados intensivos }\end{array}$ & $\begin{array}{c}48 \pm 6 \\
25 \pm 14 \\
14 \pm 4\end{array}$ & $\begin{array}{l}71 \pm 8 \\
19 \pm 3 \\
13 \pm 2\end{array}$ & $\begin{array}{l}48 \pm 5 \\
23 \pm 3 \\
11 \pm 1\end{array}$ & $\begin{array}{c}41 \pm 11 \\
19 \pm 6 \\
16 \pm 4\end{array}$ & $\begin{array}{c}47 \pm 11 \\
14 \pm 5 \\
10 \pm 6\end{array}$ & $\begin{array}{l}54 \pm 4 \\
21 \pm 2 \\
12 \pm 1\end{array}$ \\
\hline $\begin{array}{l}\text { Nascidos vivos/Instituição }+ \\
\quad<1.000 \mathrm{~g} \\
1.000-1.499 \mathrm{~g} \\
1.500-2.499 \mathrm{~g} \\
\text { Total }\end{array}$ & $\begin{array}{c}4 \pm 2 \\
6 \pm 3 \\
57 \pm 26 \\
348 \pm 99\end{array}$ & $\begin{array}{c}5 \pm 1 \\
8 \pm 2 \\
57 \pm 8 \\
434 \pm 59\end{array}$ & $\begin{array}{c}3 \pm 1 \\
5 \pm 2 \\
37 \pm 6 \\
326 \pm 33\end{array}$ & $\begin{array}{c}2 \pm 1 \\
4 \pm 0 \\
27 \pm 7 \\
351 \pm 26\end{array}$ & $\begin{array}{c}2 \pm 0 \\
2 \pm 1 \\
30 \pm 11 \\
332 \pm 123\end{array}$ & $\begin{array}{c}3 \pm 0 \\
6 \pm 1 \\
43 \pm 4 \\
365 \pm 26\end{array}$ \\
\hline $\begin{array}{l}\text { Profissionais/Instituição * } \\
\text { Pediatras diaristas } \\
\text { Pediatras plantonistas } \\
\text { Enfermeiras - Diurno } \\
\text { Enfermeiras - Noturno }\end{array}$ & $\begin{array}{c}4 \pm 1 \\
24 \pm 8 \\
4 \pm 1 \\
7 \pm 5\end{array}$ & $\begin{array}{c}0 \pm 0 \\
25 \pm 3 \\
3 \pm 1 \\
3 \pm 1\end{array}$ & $\begin{array}{c}3 \pm 1 \\
28 \pm 3 \\
4 \pm 1 \\
4 \pm 1\end{array}$ & $\begin{array}{c}3 \pm 2 \\
12 \pm 2 \\
3 \pm 0 \\
2 \pm 0\end{array}$ & $\begin{array}{c}5 \pm 3 \\
11 \pm 1 \\
5 \pm 3 \\
3 \pm 1\end{array}$ & $\begin{array}{c}3 \pm 1 \\
23 \pm 2 \\
4 \pm 1 \\
4 \pm 1\end{array}$ \\
\hline
\end{tabular}

$\mathrm{N}=$ Norte; NE = Nordeste; $\mathrm{SE}=$ Sudeste; $\mathrm{S}=\mathrm{Sul} ; \mathrm{CO}=$ Centro-Oeste.

* número médio \pm erro padrão por instituição analisada em cada região.

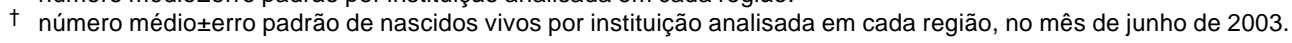


Cada maternidade dispunha, em mediana, de quatro salas de parto (variação: duas a 14) e três mesas de reanimação neonatal (variação: uma a oito). No total, as 36 instituições contavam com 125 mesas para a reanimação neonatal. Em termos de recursos materiais, todas as mesas dispunham de fonte de calor radiante, fonte de oxigênio e de vácuo e material para aspiração de vias aéreas. Quanto ao material para a ventilação, o balão auto-inflável com volume adequado estava presente em 123 (93\%) mesas e as máscaras faciais em 120 (96\%). Em termos do material para intubação traqueal, 116 (93\%) tinham laringoscópio e as cânulas apropriadas estavam presentes em mais de $90 \%$ das mesas. As principais medicações para reanimação neonatal estavam disponíveis para uso imediato em todas as mesas.

\section{Ensino da reanimação neonatal}

Das maternidades estudadas, 23 (64\%) recebiam alunos de graduação em Medicina e Enfermagem, 75\% treinavam residentes de primeiro e segundo ano de Pediatria e $42 \%$ ofereciam treinamento para especialização ou residência em Neonatologia. Com relação ao número de residentes de primeiro ano em Pediatria em 2003, os dois hospitais da região Norte que ofereciam esse treinamento contavam, no total, com duas vagas preenchidas, os oito do Nordeste com 30 residentes, os 12 do Sudeste com 121 residentes, os quatro da região Sul com 33 residentes e um da região Centro-Oeste tinha 11 residentes. Já quanto ao número de residentes (R3) ou especializandos em Neonatologia por região geográfica, seis maternidades do Nordeste, quatro do Sudeste, quatro do Sul e uma do Centro-Oeste dispunham, respectivamente, de $11,13,13$ e duas vagas preenchidas em 2003.

Das 23 instituições que recebiam alunos de graduação, cerca de metade oferecia algum treinamento formal em reanimação neonatal para alunos de Medicina, e só uma treinava alunos de Enfermagem. Já para residentes em Pediatria e especializandos ou residentes em Neonatologia, todas as que recebiam tais médicos em formação dispunham de programa de treinamento nos procedimentos necessários para reanimar o recém-nascido na sala de parto (Tabela 2).
Das 12 instituições que ofereciam treinamento em reanimação neonatal para alunos de graduação médica, em quatro era somente teórico, com um mínimo de 1 e máximo de 4 horas. Nas outras oito instituições, o treinamento era teórico-prático, com um mínimo de 8 e máximo de 34 horas. A única instituição que oferecia treinamento para alunos de graduação em Enfermagem localizava-se na região Nordeste, sendo um curso de 8 horas, apenas teórico. Para os residentes em Pediatria, quatro maternidades ministravam apenas 2 a 3 horas de aula teórica e as outras 23 contavam com um treinamento teórico-prático com duração mínima de 4 e máxima de 68 horas. Ressalta-se que, dentre as 23 instituições que ofereciam treinamento teórico-prático para residentes em Pediatria, em cinco (22\%) a duração desse treinamento era inferior a 8 horas. Para os residentes/ especializandos em Neonatologia, uma maternidade ministrava apenas 2 horas de aula teórica, e as outras 14 ofereciam treinamento teórico-prático mínimo de 3 e máximo de 68 horas. Vale frisar que, entre estas 14 últimas, em duas $(14 \%)$ a duração do treinamento teórico-prático era inferior a 8 horas.

Das 23 instituições com alunos de quinto ou sexto ano médico, em 13 os alunos participavam do atendimento ao recém-nascido na sala de parto. Dessas 13 , duas instituições não ofereciam treinamento formal em reanimação neonatal para os alunos. Das 23 instituições com alunos de terceiro ou quarto ano de Enfermagem, em oito os alunos participavam do atendimento ao recém-nascido na sala de parto, das quais sete não ofereciam treinamento formal em reanimação. Já as 27 maternidades que eram campo de atuação prática para residentes de primeiro e segundo ano de Pediatria e as 15 que eram campo de atuação prática para residentes/especializandos em Neonatologia ofereciam o treinamento teórico e/ou teórico-prático para todos esses médicos que atuavam nas salas de parto.

\section{Discussão}

Recém-nascidos com asfixia requerem reanimação apropriada em sala de parto para sobreviver ao período neonatal com o mínimo de seqüelas. Cerca de 6 a $10 \%$ dos nascidos vivos necessitam de assistência respiratória imediata, mas,

Tabela 2 - Número de instituições que ensinam reanimação neonatal dentre aquelas que se constituem em local de ensino para graduação em Medicina e Enfermagem, residência em Pediatria e residência/especialização em Neonatologia, por região geográfica

\begin{tabular}{lcccccc}
\hline & N & NE & SE & S & Co & Total \\
\hline Alunos de quinto/sexto ano médico & $2 / 3$ & $5 / 9$ & $2 / 7$ & $3 / 3$ & $0 / 1$ & $12 / 23(52 \%)$ \\
Alunos de terceiro/quarto ano Enfermagem & $0 / 2$ & $1 / 8$ & $0 / 10$ & $0 / 1$ & $0 / 2$ & $1 / 23(4 \%)$ \\
R1 ou R2 de Pediatria & $2 / 2$ & $8 / 8$ & $12 / 12$ & $4 / 4$ & $1 / 1$ & $27 / 27(100 \%)$ \\
R3 ou especializando em Neonatologia & - & $6 / 6$ & $4 / 4$ & $4 / 4$ & $1 / 1$ & $15 / 15(100 \%)$ \\
\hline
\end{tabular}

$\mathrm{N}=$ Norte $; \mathrm{NE}=$ Nordeste $\mathrm{SE}=$ Sudeste $; \mathrm{S}=\mathrm{Sul} ; \mathrm{CO}=$ Centro-Oeste . 
uma vez estabelecida a respiração espontânea, a maior parte desses bebês sobrevive sem precisar de outras medidas de suporte vital ${ }^{16-18}$. De acordo com a Organização Mundial da $\mathrm{Saúde}^{8}$, a educação e o treinamento em reanimação oferecem a possibilidade de aplicação imediata de avanços científicos recentes para diminuir a morbidade e a mortalidade neonatais.

A introdução de programas formais de reanimação neonatal parece ter tido um impacto positivo nos desfechos clínicos neonatais mais importantes, embora a maior parte dos estudos a esse respeito apresentem falhas metodológicas graves. Na China, a implementação do ensino da reanimação em um único hospital reduziu em $65 \%$ a mortalidade neonatal ${ }^{19}$. Na Índia, em 10 hospitais universitários, a introdução do Programa de Reanimação reduziu a mortalidade neonatal precoce de 31 para 24 por 1.000 nascidos vivos e a incidência de encefalopatia hipóxicoisquêmica em $70 \%{ }^{17}$. O treinamento teórico-prático em reanimação neonatal resulta também em benefícios a curto prazo para o recém-nascido, incluindo a melhora da pontuação do Boletim de Apgar ${ }^{20}$.

É nesse contexto que o presente estudo se insere, ao procurar verificar, nas maternidades públicas das capitais brasileiras, como se dá o ensino de reanimação neonatal aos médicos, pediatras, neonatologistas e enfermeiros em formação. Mas, antes de discutir a questão do ensino propriamente dita, é importante analisar se tais instituições tinham condições, em termos de recursos físicos, materiais e humanos para a reanimação neonatal, de ser campo de atuação prática para profissionais de saúde em formação.

Como pode ser observado nos resultados, os hospitais e maternidades estudados eram predominantemente públicos. O fato de $70 \%$ deles fazerem parte do Programa Iniciativa Amigo da Criança e $90 \%$ serem referência para gestação de risco indica que se tratavam de instituições com preocupação em relação ao atendimento das gestantes e de seus recém-nascidos. De modo geral, as instituições incluídas na casuística apresentavam potencial para se constituir em campo de treinamento para médicos e enfermeiros em formação. Nesse sentido, a presença nos serviços de 3\% de recém-nascidos de muito baixo peso e $15 \%$ de baixo peso, percentual mais elevado que o da população brasileira (respectivamente, 1 e $8 \%$, em 2001) ${ }^{1}$, indica que a necessidade de procedimentos de reanimação neonatal era usual nas instituições analisadas, uma vez que 90 a $100 \%$ dos pacientes com peso ao nascer abaixo de $1.500 \mathrm{~g}$ necessitam de alguma manobra de reanimação em sala de parto ${ }^{21-23}$. Em termos de recursos humanos, a presença de pediatras e enfermeiros contratados para cuidar do recém-nascido nas salas de parto indica que o aluno e o residente, quando atuantes nessas instituições, podem, de fato, aprender, em vez de assumir um papel assistencial sem possuir qualificação para tal. Finalmente, observa-se que o material básico para o atendimento do neonato estava disponível em praticamente todas as mesas de reanimação de cada instituição. Dessa forma, condições mínimas para que alunos de graduação e residentes aprendam em serviço estavam disponíveis nas instituições analisadas.
Assim, se os hospitais estudados se constituíam, em porcentagem expressiva, em campo de atividade prática para alunos e residentes e diante da disponibilidade, de modo geral, de recursos físicos, materiais e humanos para o ensino da reanimação neonatal, surge a pergunta: será que os hospitais com alunos e residentes estavam ensinando a esses (futuros) profissionais de saúde os fundamentos práticos da reanimação do recém-nascido em sala de parto? Diante dessa questão, vale ressaltar que, tradicionalmente, o treinamento nos procedimentos de reanimação era executado com a observação, por profissionais em formação, da atuação de um especialista na prática clínica. Gradualmente, os mais jovens iam assumindo responsabilidade progressiva para a execução dos procedimentos em pacientes reais. Esses profissionais, por sua vez, passavam a ensinar os mais jovens, fechando uma cadeia circular de formação. A eficácia desse modelo dependia da repetição da exposição dos mais jovens às diferentes situações clínicas que requerem intervenção e da habilidade e competência docente dos mais velhos ${ }^{24}$. Nos últimos anos, o aprendizado em medidas de suporte vital vem mudando de maneira progressiva, com a concretização do chamado ensino "prático", em que os alunos treinam os procedimentos em manequins e simulam situações reais, complementando o ensino tradicional. Essa estratégia capacita o aluno a levar a cabo manobras de reanimação de forma integrada e em seqüência racional, a sentir-se capaz de aplicá-las na vida real e proporciona educação continuada, atualizando o conhecimento dos profissionais que prestam assistência ao recém-nascido na sala de parto 22 .

Em termos de graduação médica, das 23 maternidades que recebiam alunos de quinto e sexto ano, apenas $52 \%$ delas ofereciam algum treinamento em reanimação. Ainda sem levar em conta o tipo de ensino de reanimação oferecido, essa situação é preocupante, pois significa que um percentual importante de médicos está sendo formado sem conceitos básicos em relação a um procedimento estratégico para reduzir a mortalidade neonatal. Se tais profissionais entrarem no mercado de trabalho sem cursar programas de especialização, os seus recursos para reanimar recém-nascidos dependerão apenas da exposição prévia a essa situação prática na graduação, que pode variar extremamente, de acordo com o currículo, o campo de treinamento e a capacidade docente. Quando consideramos que o aluno de Medicina é o que mais incorpora os conhecimentos ministrados nos cursos teórico-práticos de reanimação neonatal ${ }^{25}$, pode-se ter uma dimensão da oportunidade perdida. Essa observação é reforçada ainda pela constatação, no presente estudo, de que um terço daquelas instituições que oferecem treinamento em reanimação neonatal aos seus alunos de graduação médica, o fazem de forma exclusivamente teórica. Está se perdendo uma oportunidade ímpar de expandir o conhecimento das técnicas de reanimação neonatal a todos os médicos deste país e de fazer com que se crie uma consciência crítica da prioridade dessa ação estratégica na prevenção de milhares de mortes de recém-nascidos ao ano no Brasil ${ }^{1,2}$ e de milhões no mundo $0^{4,8}$. 
O retrato do ensino para graduação em Enfermagem, nessas instituições, é ainda pior. Das 23 maternidades que diziam receber alunos de terceiro e quarto ano de Enfermagem, apenas uma referiu ministrar algum treinamento em reanimação neonatal, sendo que era apenas teórico. De acordo com os programas internacionais, as enfermeiras podem e devem ser treinadas para o atendimento ao recém-nascido em sala de parto para auxiliarem o pediatra ou, na ausência deste, conduzirem os procedimentos iniciais de reanimação neonatal26,27. No Brasil, as enfermeiras podem realizar os passos iniciais da reanimação e a ventilação com balão e máscara, além de auxiliarem os pediatras na massagem, intubação e uso de medicações $^{28}$. O treinamento dos alunos de enfermagem nos procedimentos básicos em reanimação, conforme orientação da Sociedade Brasileira de Pediatria ${ }^{28}$, poderia contribuir para diminuir a morbidade e a mortalidade por asfixia perinatal em nosso meio.

A situação é mais animadora no que se refere aos médicos que estão cursando especialização em Pediatria e Neonatologia. Para esses profissionais, todas as instituições que os recebiam dispunham de programa de treinamento específico. O tipo de treinamento oferecido também estava mais de acordo com as considerações que valorizam o aprendizado prático em simulações de situações reais. Nota-se, entretanto, que em $20 \%$ das maternidades o número de horas de treinamento teóricoprático para pediatras e neonatologistas em formação era inferior ao mínimo de 8 horas preconizado pela Sociedade Brasileira de Pediatria.

Dessa forma, os resultados obtidos indicam que o ensino de reanimação neonatal por meio de cursos teórico-práticos formais é insuficiente para os alunos de graduação em Medicina e Enfermagem e heterogêneo para os residentes em Pediatria e em Neonatologia.

\section{Agradecimentos}

Agradecemos o Grupo Executivo em Reanimação Neonatal da Sociedade Brasileira de Pediatria, gestão 2001 a 2003, por ajudar a viabilizar a concretização de todo o projeto de pesquisa, no qual se insere este artigo.

\section{Pesquisadores do Programa de Reanimação Neonatal da Sociedade Brasileira de Pediatria (SBP)}

Região Norte - Rossiclei de Souza Pinheiro (Maternidade Balbina Mestrinho, Manaus, AM), Vânia Cecília da Silva Pinto (Santa Casa do Pará, Belém, PA), Elaine A. das N. Figueiredo (Hospital de Clínicas Gaspar Viana, Belém, PA).

Região Nordeste - Vanda Maria Ferreira Simões (Hospital Universitário, São Luís, MA), Maria de Fátima A. Carvalho (Maternidade Marly Sarney, São Luís, MA), Luiz Edson dos Santos Costa (Maternidade Dona Evangelina Rosa, Teresina, PI), Luiz Carlos Batista de Souza (Materni- dade Assis Chateaubriand, Fortaleza CE), Dilma Veras Leal (Hospital Geral de Fortaleza, CE), Mayra Isabel Correia Pinheiro (Hospital Geral Dr. César Cals, Fortaleza, CE), Rosa Maria Vaz dos Santos (Maternidade Januário Cicco, Natal, RN), Cláudia Roberta M. Pereira (Hospital Agamenom Magalhães, Recife, PE), Ana Claire Pimenteira Thomaz (Hospital Universitário Prof. Alberto Antunes, Maceió, AL), Carline Rabelo de Oliveira (Maternidade Hildete Falcão Baptista, Aracajú, SE), Tereza Paim Xavier (Instituto de Perinatologia da Bahia, Salvador, BA).

Região Sudeste - Marina Trópia G. Guerzoni (Hospital das Clínicas, Belo Horizonte, MG), Siura Aparecida Borges Silva (Maternidade Odete Valadares, Belo Horizonte, MG), Valmin Ramos da Silva (Associação Beneficente Pró-Matre de Vitória, ES), José Dias Rego (Hospital Maternidade Alexander Fleming, Rio de Janeiro, RJ), Antonio Carlos de A. Melo (Maternidade Carmela Dutra, Rio de Janeiro, RJ), José Vicente Vasconcelos (Maternidade Leila Diniz, Rio de Janeiro, RJ), Arnaldo Costa Bueno (Maternidade Oswaldo Nazareth, Rio de Janeiro, RJ), Ana Maria A G. P. de Melo (Hospital Municipal Dr. Alípio Corrêa Netto, São Paulo, SP), Bettina Barbosa D. Figueira (Hospital Municipal Dr. Cármino Caricchio, São Paulo, SP), Cheung Hei Lee Russo (Hospital Municipal Dr. Fernando M. P. Rocha, São Paulo, SP), Cláudia Tanuri (Hospital Municipal Dr. Mário M. A. Silva, São Paulo, SP), Marina da Rosa Faria (Conjunto Hospitalar do Mandaqui, São Paulo, SP), Vera Lucia Figueiredo Senise (Hospital Ipiranga, São Paulo, SP), Glória Celeste V. R. Fernandes (Hospital Maternidade Leonor M. de Barros, São Paulo, SP).

Região Centro-Oeste - José Mendes de Carvalho Filho (Santa Casa de Campo Grande, MS), Maria Bárbara F. Gomes (Maternidade Nascer Cidadão, Goiânia, GO), Aída Magalhães Gomes (Hospital Regional de Taguatinga, Brasília, DF).

Região Sul - Luvercy Rodrigues Filho (Clínica e Maternidade Nossa Sra. do Rosário, Curitiba, PR), Evanguelia Athanasio Shwetz (Hospital Universitário Evangélico, Curitiba, PR), Leila Denise Cesário Pereira (Maternidade Carmela Dutra, Florianópolis, SC), Célia Boff de Magalhães (Santa Casa de Misericórdia de Porto Alegre, RS), Ivana Santos Varella (Hosp. Nossa Sra. da Conceição, Porto Alegre, RS).

\section{Referências}

1. Ministério da Saúde - Fundação Nacional de Saúde [homepage na Internet]. Brasília: Nascidos vivos - Brasil. 2000-2002. Disponível em: URL:http//datasus.gov.br

2. Ministério da Saúde - Fundação Nacional de Saúde. [homepage na Internet\}. Brasília: Indicadores Demográficos - Brasil. 2000 e 2001. Disponível em: URL:http//datasus.gov.br

3. Ministério da Saúde - Fundação Nacional de Saúde. [homepage na Internet]. Brasília: Mortalidade infantil. 2001. Disponível em: URL:http//www.funasa.gov.br

4. Jones J, Steketee RW, Black RE, Bhutta ZA, Morris SS, Bellagio Child Survival Study Group. How many child deaths can we prevent this year? Lancet. 2003;362:65-71.

5. Xiaoyu Z. Neonatal resuscitation. World Health Forum. 1993; $14: 289-90$

6. Bhatia BD, Bhat BV, Dey AK, Mohan PV. Training of final year MBBS students in neonatal resuscitation. Indian Pediatr. 1993;30:113-5. 
7. Maibach EW, Schieber RA, Carroll MF. Self-efficacy in pediatric resuscitation: implications for education and performance. Pediatrics. 1996;97:94-9.

8. WHO/FRH/MSM/96.13 Distr. General Division of Reproductive Health (Technical Support) Maternal and Newborn Health/Safe Motherhood. Essential Newborn Care Report of a Technical Working Group. Trieste 1994;25-29A.

9. Kattwinkel J, Niermeyer S, Nadkarni V, Tibballs J, Phillips B, Zideman $D$, et al. Resuscitation of the newly born infant - an advisory statement from the pediatric working group of the International Liaison Committee on Resuscitation. Circulation. 1999;99:1927-38.

10. Kattwinkel J. Textbook of Neonatal Resuscitation. 4th ed. Chicago: American Academy of Pediatrics/American Heart Association; 2000.

11. American Academy of Pediatrics. Neonatal resuscitation program international activity report - September 2002. American Academy of Pediatrics 2002 National Conference and Exhibition; 2002 October 18; Boston-MA, USA.

12. Almeida MFB, Guinsburg R. Histórico dos cursos de treinamento para a reanimação neonatal. In: Rego JD, editor. Reanimação neonatal. São Paulo: Atheneu; 2004. p. 173-8.

13. Sociedade Brasileira de Pediatria. Programa de Reanimação Neonatal. 2004. Comunicação pessoal.

14. Ministério da Saúde. Brasil. Recursos humanos e material mínimo para assistência ao RN na sala de parto. Portaria SAS/ MS No 96, 1994.

15. Ministério da Saúde. Secretaria de políticas de saúde. Área técnica de saúde da mulher. Parto, aborto e puerpério: assistência humanizada à mulher. Brasília: Ministério da Saúde, 2001.

16. Perlman JM, Risser R. Cardiopulmonary resuscitation in the delivery room. Associated clinical events. Arch Pediatr Adolesc Med. $1995 ; 149: 20-25$

17. Deorari AK, Singh M, Paul VK, Vidyasagar D, The Medical Colleges Network. Impact of education and training on neonatal resuscitation practices in 14 teaching hospitals in India. Ann Trop Pediatr. 2001;21:29-33.

18. Singhal N, McMillan DD, Yee WH, Akierman AR, Yee YJ. Evaluation of the effectiveness of the standardized Neonatal Resuscitation Program. J Perinatol. 2001;21:388-92.

19. Zhu XY, Fang HQ, Zeng SP, Li YM, Lin HL, Shi SZ. The impact of the neonatal resuscitation program guidelines (NRPG) on the neonatal mortality in a hospital in Zhuhai, China. Singapore Med J. $1997 ; 38: 485-7$
20. Patel D, Piotrowski ZH, Nelson MR, Sabich R. Effect of a statewide neonatal resuscitation training on Apgar scores among high-risk neonates in Illinois. Pediatrics. 2001;107:648-55.

21. Oliveira ACA, Draque CM, Almeida MFB, Guinsburg R, Miyoshi $\mathrm{MH}$, Goulart AL, et al. Eficácia das manobras de reanimação em recém-nascidos pré-termo. Anais do XVI Congresso Brasileiro de Perinatologia e XIII Reunião de Enfermagem Perinatal; 1820 novembro 1998; Salvador-BA, Brasil: Anais, 1998:268.

22. Grupo de Reanimacón Neonatal de la Sociedad Española de Neonatologia. Cursos de reanimación cardiopulmonar neonatal. Ann Pediatr. 2003;58:252-6.

23. Rede Brasileira de Pesquisas Neonatais. Uso antenatal de corticosteróide e condições de nascimento de pré-termos nos hospitais da rede brasileira de pesquisas neonatais. Rev Bras Ginecol Obstetr. 2004;26:177-82.

24. Hall JG. See one, do one, teach one. Pediatrics. 1999;103:155-6.

25. Almeida MFB, Guinsburg R, Costa JO, Freire LM and Instructors of Neonatal Resuscitation Program. Brazilian neonatal resuscitation program: factors that interfere with knowledge gain after training. J Perinatol. 2002;22:608-9.

26. Britton JR. Neonatal nurse practitioner and physician use on a newborn resuscitation team in a community hospital. J Pediatr Health Care. 1997;11:61-5.

27. Halamek LP, Kaegi DM, Gaba DM, Sowb YA, Smith BC, Smith BE, et al. Time for a new paradigm in pediatric medical education: teaching neonatal resuscitation in a simulated delivery room environment. Pediatrics. 2000;106:1-6.

28. Sociedade Basileira de Pediatria. Manual para auxiliares da reanimação. Rio de Janeiro: SBP; 2002.

Correspondência:

Ruth Guinsburg

Rua Vicente Felix, 77/09

CEP 01410-000 - São Paulo, SP

Telefax: (11) 5579.1676 / 5579.4982

E-mail: ruthgbr@netpoint.com.br 